PROCEEDINGS OF THE

AMERICAN MATHEMATICAL SOCIETY

Volume 126, Number 4, April 1998, Pages 1253-1255

S 0002-9939(98)04280-4

\title{
TORI IN CERTAIN ASPHERICAL FOUR-MANIFOLDS
}

\author{
ALLAN L. EDMONDS
}

(Communicated by Ronald A. Fintushel)

\begin{abstract}
The homology classes represented by embedded or mapped tori in the product of two surfaces are completely characterized.
\end{abstract}

If $X^{4}$ is a 4-manifold, then any element $\xi \in H_{2}\left(X^{4}\right)$ can be represented by a map from a closed, oriented surface, and also by an embedded, closed, oriented surface (perhaps of bigger genus). If $X^{4}$ is simply connected then $H_{2}\left(X^{4}\right) \approx \pi_{2}\left(X^{4}\right)$ by the Hurewicz Theorem, so that every class $\xi$ is represented by a map $S^{2} \rightarrow X^{4}$. It has been an intensively studied fundamental problem to determine which classes are represented by smooth or topologically embedded 2 -spheres, and, more generally, to determine the minimum genus of an embedded surface representing $\xi$. In general there are striking differences between the smooth and topological categories. See, for example, [2] and [3] for some of the latest results in this area.

In this note we begin to address similar questions in the aspherical case, in which there are no interesting 2 -spheres at all, apart from "local knot theory."

We determine which 2-dimensional homology classes can be represented by embedded tori in $X^{4}=F^{2} \times G^{2}$, where $F^{2}$ and $G^{2}$ are closed orientable surfaces of genus greater than 1 . It turns out that there is no difference between the topological and differentiable categories in this case.

Theorem 1. Let $F^{2}$ and $G^{2}$ be closed orientable surfaces of genus greater than 1. An element $\xi \in H_{2}\left(F^{2} \times G^{2}\right)$ is represented by a topological or differentiable embedded torus $T^{2} \subset F^{2} \times G^{2}$ if and only if $\xi=\alpha \times \beta$ for some $\alpha \in H_{1}\left(F^{2}\right)$ and $\beta \in H_{1}\left(G^{2}\right)$.

This follows immediately from the following two more precise propositions.

Proposition 1. Any map $f: T^{2} \rightarrow F^{2} \times G^{2}$ represents a homology class of the form $\alpha \times \beta$ for some $\alpha \in H_{1}\left(F^{2}\right)$ and $\beta \in H_{1}\left(G^{2}\right)$.

Proposition 2. If $\alpha \in H_{1}\left(F^{2}\right)$ and $\beta \in H_{1}\left(G^{2}\right)$ are given, then the homology class $\alpha \times \beta$ is represented by a smooth embedding $f: T^{2} \rightarrow F^{2} \times G^{2}$.

Lemma 1. If $F^{2}$ is a surface of negative euler characteristic, then any two commuting elements in $\pi_{1}\left(F^{2}\right)$ generate a cyclic subgroup of $\pi_{1}\left(F^{2}\right)$.

Proof. Let $\alpha, \beta \in \pi_{1}\left(F^{2}\right)$ be commuting elements and let $S \rightarrow F^{2}$ be the covering map, where $\pi_{1}(S)$ corresponds to the subgroup of $\pi_{1}\left(F^{2}\right)$ generated by $\alpha$ and $\beta$. If it is a finite-sheeted covering, then $S \cong T^{2}$ by the classification of surfaces, and

Received by the editors October 7, 1996.

1991 Mathematics Subject Classification. Primary 57N13, 57N35.

(C)1998 American Mathematical Society 
hence $\chi(S)=0$. But multiplicativity of the euler characteristic then implies that $\chi\left(F^{2}\right)=0$ too, a contradiction. Therefore the covering must be an infinite-sheeted covering. In particular, $S$ is non-compact, and hence has a free fundamental group. Since $\pi_{1}(S)$ is abelian, this implies that it is cyclic, as required.

Corollary 1. If $F^{2}$ is a closed orientable surface of negative euler characteristic, and $f: T^{2} \rightarrow F^{2}$ is a map, then there is a primitive homology class $\alpha \in H_{1}\left(F^{2}\right)$ such that the image of $f_{*}: H_{1}\left(T^{2}\right) \rightarrow H_{1}\left(F^{2}\right)$ is contained in the cyclic subgroup generated by $\alpha$.

Proof. Since $\pi_{1}\left(T^{2}\right) \approx H_{1}\left(T^{2}\right)$, the image of $H_{1}\left(T^{2}\right)$ in $H_{1}\left(F^{2}\right)$ is the image under the Hurewicz homorphism of the image of $\pi_{1}\left(T^{2}\right)$ in $\pi_{1}\left(F^{2}\right)$, which is cyclic by Lemma 1, and hence contained in a cyclic subgroup generated by a primitive element.

We will need the following well-known result. For a proof, see [4].

Lemma 2. If $\alpha \in H_{1}\left(F^{2}\right)$ is a nonzero homology class, then $\alpha$ is represented by a simple closed curve embedded in $F^{2}$ if and only if $\alpha$ is primitive.

Proof of Proposition 1. Let $f: T^{2} \rightarrow F^{2} \times G^{2}$ represent a nonzero homology class and let $p_{F}: F^{2} \times G^{2} \rightarrow F^{2}$ and $p_{G}: F^{2} \times G^{2} \rightarrow G^{2}$ denote the projections onto the two factors. By Corollary 1 the image of $\left(p_{F} \circ f\right)_{*}: H_{1}\left(T^{2}\right) \rightarrow H_{1}\left(F^{2}\right)$ is contained in a cyclic subgroup $\langle\alpha\rangle$ generated by a primitive element $\alpha \in H_{1}\left(F^{2}\right)$. Similarly, the image of $\left(p_{G} \circ f\right)_{*}: H_{1}\left(T^{2}\right) \rightarrow H_{1}\left(G^{2}\right)$ is contained in a cyclic subgroup $\langle\beta\rangle$ generated by a primitive element $\beta \in H_{1}\left(G^{2}\right)$. Now by Lemma 2 both $\alpha$ and $\beta$ are represented by simple closed curves $A$ and $B$ in $F^{2}$ and $G^{2}$, respectively. It follows that $f_{*}\left[T^{2}\right]$ is a multiple of $[A \times B]$ in $H_{2}\left(F^{2} \times G^{2}\right)$. But $n(\alpha \times \beta)=(n \alpha) \times \beta$, which is what is needed.

Proof of Proposition 2. If we write $\alpha=m \alpha^{*}$ and $\beta=n \beta^{*}$, where $\alpha^{*}$ and $\beta^{*}$ are primitive homology classes, then $\alpha \times \beta=m n \alpha^{*} \times \beta^{*}=\left(m n \alpha^{*}\right) \times \beta^{*}$. Now, by Lemma $2, \alpha^{*}$ and $\beta^{*}$ can be represented by simple closed curves $A^{*}$ and $B^{*}$, respectively. Since $A^{*}$ and $B^{*}$ have trivial normal bundles, so does the torus $A^{*} \times$ $B^{*} \subset F^{2} \times G^{2}$. The proof then is completed by Lemma 3 below.

Lemma 3. The map $T^{2} \rightarrow T^{2} \times D^{2}$ given by $(z, w) \mapsto\left(z^{k}, w, 0\right)$ is homotopic to an embedding.

Proof. Just define an explicit embedding by $(z, w) \mapsto\left(z^{k}, w, z\right)$

Remark 1. Lemma 3 generalizes to state that if $F^{2}$ is a closed oriented surface of genus $g$ and $n$ is a positive integer, then the homology class in $H_{2}\left(F^{2} \times D^{2}\right)$ corresponding to $n\left[F^{2} \times\{0\}\right]$ is represented by an embedding of the surface of genus $n g-(n-1)$. This is the least possible genus that can even represent $n\left[F^{2} \times\{0\}\right]$ by a map, by Kneser's inequality. For a modern exposition of Kneser's theorem, see [5].

Remark 2. Any map of nonzero degree $T^{2} \rightarrow T^{2}$ is homotopic to a covering, and any covering $T^{2} \rightarrow T^{2} \times\{0\} \subset T^{2} \times D^{2}$ is homotopic to an embedding. The proof is somewhat more difficult than that of Lemma 3 and depends on lifting a pair of commuting permutations $\sigma, \tau \in S_{n}$, that generate a transitive subgroup of $S_{n}$, to a pair of commuting braids $b_{\sigma}$ and $b_{\tau}$ over them in the braid group $B_{n}$. 
Remark 3. No nontrivial connected covering $F^{2}$ of the 0-section of a nontrivial oriented 2-disk bundle $E$ over a closed orientable surface $G^{2}$ is homotopic to an embedding of $F^{2}$ in $E$. See [1], for example.

It follows from the results of this note that a map $T^{2} \rightarrow F^{2} \times G^{2}$ is homologous to an embedding $T^{2} \rightarrow F^{2} \times G^{2}$. Is it possible that a map $T^{2} \rightarrow F^{2} \times G^{2}$ is actually homotopic to an embedding? It seems unlikely.

\section{REFERENCES}

1. A. L. Edmonds, Embedding coverings in bundles, Indiana University preprint, 1996.

2. P. B. Kronheimer and T. S. Mrowka, The genus of embedded surfaces in the projective plane, Math. Research Letters 1 (1994), 797-808. MR 96a:57073

3. Ronnie Lee and Dariusz Wilczyński, Representing homology classes by locally flat surfaces of minimum genus, preprint (1995)

4. M. D. Meyerson, Representing homology classes of closed orientable surfaces, Proc. Amer. Math. Soc. 61 (1976), 181-182. MR 54:13916

5. R. Skora, The degree of a map between surfaces, Math. Ann. 276 (1987), 415-423. MR 88f: 57018

Department of Mathematics, Indiana University, Bloomington, Indiana 47405

E-mail address: edmonds@indiana.edu 